\title{
Calcification Detection of Coronary Artery Disease in Intravascular Ultrasound Image: Deep Feature Learning Approach
}

\author{
Hannah Sofian ${ }^{1,2 *}$, Joel Chia Ming Than ${ }^{1}$, Suraya Mohammad ${ }^{3}$, \\ Norliza Mohd. Noor ${ }^{1}$
}

${ }^{1}$ Razak Faculty of Technology and Information, Universiti Teknologi Malaysia, Jalan Sultan Petra Yahya, Kuala Lumpur, 53100, MALAYSIA

${ }^{2}$ Univeristi Kuala Lumpur Malaysia Institute of Information Technology, Universiti Kuala Lumpur Jalan Sultan Ismail, Kuala Lumpur, 50250, MALAYSIA

${ }^{3}$ Univeristi Kuala Lumpur British Malaysia Institute, Universiti Kuala Lumpur

Jalan Sungai Pusu, Kuala Lumpur, 54100, MALAYSIA

* Corresponding Author

DOI: https://doi.org/10.30880/ijie.2018.10.07.005

Received 29 October 2018; Accepted 15 November 2018; Available online 30 November 2018

\begin{abstract}
Coronary artery disease (CAD) is part of the non-communicable disease (NCD) in cardiovascular disease (CVD). The blood vessel area became narrow when the calcification with the plaque embedded in the coronary artery inner wall. The radiologists and medical practitioners used visual inspection to detect calcification on IVUS image. The presence of calcification will not be able to do the measurement to calculate the maximum diameter and the maximum area for the patient coronary artery either before treatment or after treatment. More than 100 frames per patient is needed to analyse the location of the calcification. In this study, our aim is to detect the presence and the absence of the calcification in the coronary artery using intravascular ultrasound (IVUS) images with catheter frequency of $20 \mathrm{MHz}$. The IVUS images used were the original Cartesian coordinate image and the polar reconstructed coordinate image. In this study, three types of convolutional neural network (CNN) using Directed Acyclic Graph networks, were used together with five types of classifiers. The dataset used to demonstrate our framework is Dataset B from MICCAI Challenge 2011 that consists of 2175 coronary artery disease IVUS image where 530 are IVUS images with calcification and 1645 are IVUS images without calcification. The cross validation for testing and training, the k-fold value used was 2, 3, 5 and 10. The performance measures for the ResNet-50, the ResNet-101 and the Inception-V3 model shows an excellent result using support vector machine classifier and discriminant analysis for both types of images. A better improvement using polar reconstructed coordinate image when using decision tree classifier and Naïve Bayes classifier whilst ResNet-101 architecture shows an excellent performance measure when applying images polar reconstructed images when using k-nearest neighbor classifier. However, Naïve Bayes classifier has an excellent result when using Inception-V3 architecture.
\end{abstract}

Keywords: Coronary Artery Disease, Calcification, Intravascular Ultrasound, Deep learning

\section{Introduction}

Coronary artery disease is one type of cardiovascular disease (CVD). According to the World Health Organisation (WHO), 31\% of non-communicable disease (NCD) is from CVD. The build-up of lipids, plaques and calcification that embed in the inner wall of the artery that may result in narrowing the blood vessel. Therefore, the volume of blood flow is decreased. Moreover, the ruptured plaques and calcification may block the small arteries where the patient can get 
angina pectoris and finally, myocardial infarction. This calcification is an artery disease, called coronary artery calcification. Fig. 1 shows the samples of a coronary artery disease of the IVUS images. The coronary artery disease with plaques is shown in Fig. 1a where the yellow arrows indicate the plaque build-up in the artery wall whilst the calcification (red arrows) was located near to the tunica intima as shown in Fig. 1b. The IVUS catheter used ultrasound to get the reflection from the artery wall. Since the ultrasound cannot penetrate the conductor such as calcium, the IVUS image has shadow artefact behind the calcification as shown by the cyan arrow in Fig. 1b. The presence of the shadow artefact mentioned by (Pu et al., 2012) and (Jodas, Pereira, \& Tavares, 2017) indicates that the calcification is present.

In the deep learning, the series network was introduced for instance by LeNet model by (Yann LeCun, Leon Bettou, Yoshua Bengio, 1998), AlexNet model by (Krizhevsky, Sutskever, \& Hinton, 2012), VGG16 and VGG19 model by (Simonyan, 2013). Image net competition that is known as the ImageNet Large Scale Visual Recognition Challenge (ILSVRC) classification started since 2010 for $2 \mathrm{D}$ images to find the best algorithm with less error. The Direct Acyclic Graph (DAG) architectures were developed based on the series network architecture to get better performance measures result such as Inception model and ResNet model. Both models show a better result with less error compared to the series network. ResNet model had used to detect calcification for instance stroke, to detect calcification in the artery using CT (Bortsova et al., 2017), for coronary artery disease to detect calcification using intravascular ultrasound images for instaance (Sofian, Than, Ming, Mohamad, \& Noor, 2018) and for coronary artery disease to detect plaque using OCT (Gessert et al., 2018). Other than detecting calcification, ResNet model was used for breast cancer to screen the abnormality of mammogram using mammography screen test images (Xi, Shu, \& Goubran, 2018). Inception models had also been used to detect coronary artery tissue such as using Optical Coherence Tomography images (Bdolmanafi et al., 2018).

In this study, we focus on detecting calcification in IVUS images using a very deep feature learning approach. Three types of convolutional neural networks (CNN) using Directed Acyclic Graph (DAG) network, namely ResNet-50, ResNet-101 and Inception-V3 were used together with the five types of classifiers namely, Support Vector Machine classifier, Discriminant Analysis classifier, Decision Tree classifier, K-nearest Neighbour classifier and Naïve Bayes classifier.

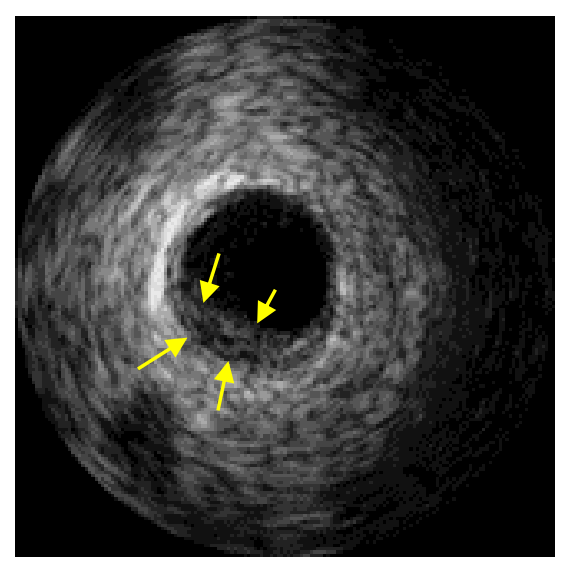

(a)

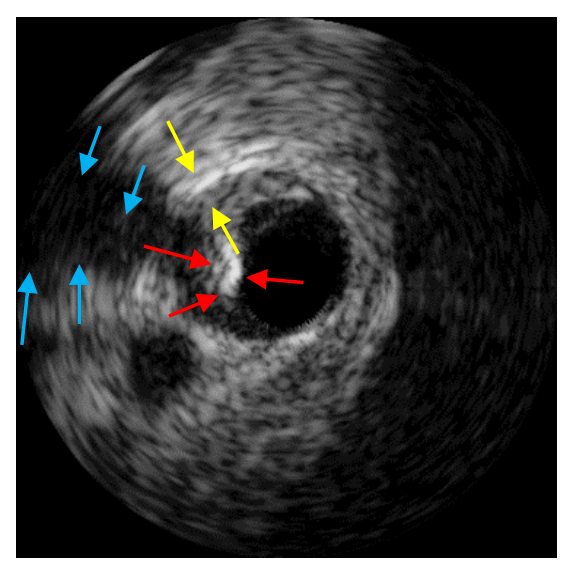

(b)

Fig. 1 - The IVUS image. (a) The plaques (The yellow arrows); (b) The plaques (The yellow arrows), shadow artefact (The cyan arrows) and calcification (The red arrows).

\subsection{Related Work}

The previous studies for the calcification detection for IVUS image was done in many ways such as (Sofian, Ng, Than, Mohamad, \& Noor, 2017) using Otsu threshold, morphological operation and empirical threshold while (Gao et al., 2015) used Rayleigh mixture model (RMM), Markov random field (MRF) to detect coronary artery boundaries and the graph searching method to detect calcification. Random walk framework was used by (Katouzian et al., 2012) to detect calcification, while (Unal et al., 2008) used shape-driven to detect calcification. (Taki et al., 2008) used parametric deformable models and the geometric deformable models to detect coronary artery boundaries and Bayesian classifier to detect calcification in coronary artery disease whereas (Filho et al., 2007) used adaptive thresholding to detect the calcification. However, the overall performance measures of all these methods to detect the calcification were about $80 \%$ - $95 \%$ for specificity and sensitivity. The study will compare the calcification detection performance between the original 
image, which is the Cartesian coordinate image and the transformed image, which are the polar reconstructed coordinate image. The IVUS samples with calcification present (The blue arrows) with the artefacts (The red arrows) are shown in Figure 2 for Cartesian coordinate image and its corresponding polar reconstructed coordinate image, respectively.

The deep learning approached was introduced by (Yann LeCun, Leon Bettou, Yoshua Bengio, 1998) called the LeNet. A competition for deep learning which is called The ImageNet Large Scale Visual Recognition Challenge was started in 2010 won by AlexNet model by (Krizhevsky, Sutskever, \& Hinton, 2012). This challenge is continuing every year to get better performance with a low error where the authors have to perform using the image classification tasks of 1000 categories with a fixed dataset that has 1.2 million training images followed with a 50k validation images and $100 \mathrm{k}$ test images. In 2013, VGG16 and VGG19 were developed by (Simonyan, 2013). In 2014, (Szegedy et al., 2014) developed GoogleNet which also known as Inception-1, followed by ResNet model in 2015 developed by (He, Zhang, Ren, \& Sun, 2015). In 2016, the Inception Models was developed by (Szegedy, Ioffe, Vanhoucke, \& Alemi, 2016) based on GoogLeNet architecture.

In this study, in detecting calcification present and absent, we applied three types of CNNs model; ResNet-50, Resnet-101 and Inception-V3 using five types of classifiers; support vector machine classifier, discriminant analysis classifier, decision tree classifier, k-nearest neighbours classifier and Naïve Bayes classifier. For performance measures validation, we used cross-validation $\mathrm{k}$-fold (where $\mathrm{k}=2,3,5$ and 10).

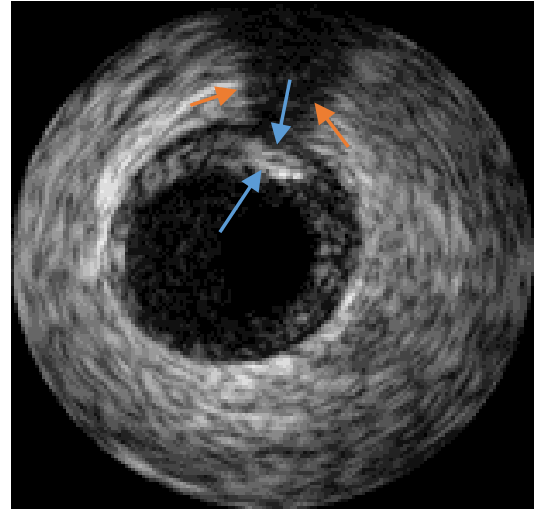

(a)

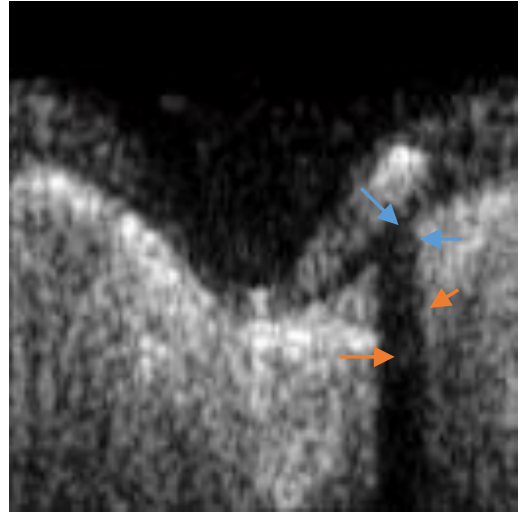

(b)

Fig. 2 - The types of IVUS images. (a) The Cartesian Coordinate (CC) Image; (b) The Polar Reconstructed Coordinate (PRC) Image.

\section{Methodology}

\subsection{Data Used in This Study}

We used Dataset B from MICCAI Challenge 2011 by (Balocco et al., 2014). This dataset consists of 2175 images extracted from in vivo pullbacks of human coronary arteries, 530 are images with calcification present, and 1645 are images with calcification absent from 10 patients. The imaging system used for the acquisition is Si5 equipped with 20 $\mathrm{MHz}$ Eagle Eye monorail catheter. All the 2175 images from the original image were transformed into their corresponding polar reconstructed image as shown in Fig. 2. The first image was the original as shown in Fig. 2(a) and the second image was the transformed polar reconstructed coordinate from its original image as shown in Fig. 2(b). We used the Daugman rubber sheet normalisation model (Daugman, 1993) to assigns each point in the tunica media, the tunica adventitia and tunica intima. The real coordinates pair coordinates unit is $(r, \theta)$ where the unit interval of $r$ is between value zero and one [0,1], and $\theta$ is the usual angular quantity that is cyclic between zero and $2 \pi[0,2 \pi]$. The Daugman rubber sheet normalisation was used to transform the Cartesian image to polar reconstructed coordinate image. The IVUS region image is: $\mathrm{I}(\mathrm{x}, \mathrm{y})$, the original Cartesian coordinates are: $(\mathrm{x}, \mathrm{y})$ and the normalised polar reconstructed coordinates are: $(\mathrm{r}, \theta)$. The coordinates IVUS boundaries along the $\theta$ direction are $\mathrm{x}_{0}, \mathrm{y}_{0}$ and $\mathrm{x}, \mathrm{y}$.

\subsection{Directed Acyclic Graph (DAG) networks}

This study used deep feature learning approach based on directed acyclic graph (DAG) networks. We used two types of models that were ResNet and Inception. ResNet has two architectures we applied, ResNet-50 architecture and ResNet101 architecture whilst Inception model, we only used Inception-V3 architecture. In this study, we used three types of architectures, ResNet-50 architectures, ResNet-101 architectures and Inception-V3 architectures. The two types of 
images chose were Cartesian coordinate image and polar reconstructed coordinate image, then, compared using different types of classifiers and getting the outcome results using performance measures result.

\subsubsection{ResNet Architectures}

There are five types of ResNet models which are ResNet18, ResNet34, ResNet50. ResNet101 and ResNet152 as shown in Table 1. In this study, we used ResNet-50 as shown in column six (The cyan outline) and ResNet101 in column seven (The brown outlined) shown in Table 1. ResNet-50, ResNet101 and ResNet-152 used the bottleneck building block as shown in Figure 3 to add more layers. The difference between ResNet-50, ResNet-101 and ResNet-152 were at the conv_4 (The horizontal navy blue outlined). Using the bottleneck building block as shown in Figure 4, the ResNet-50 add another six layers, ResNet-101 add another 23 layers, and ResNet-152 used to add another 36 layers. We analysed the two types of images using ResNet-50 architecture and ResNet-101 architecture using fc1000 layer at row seven as shown in Table 1.

Table 1 - The five types of ResNet from left 18-layers, 34-layers, 50-layers, 101-layers and 152-layers [22].

\begin{tabular}{|c|c|c|c|c|c|c|c|c|c|}
\hline $\begin{array}{l}\text { Layer } \\
\text { name }\end{array}$ & $\begin{array}{c}\text { Out } \\
\text { put size }\end{array}$ & 18-layer & 34-layer & \multicolumn{2}{|l|}{ 50_layer } & \multicolumn{2}{|l|}{ 101-layer } & \multicolumn{2}{|l|}{ 152-layer } \\
\hline Conv_1 & $112 \times 112$ & \multicolumn{2}{|l|}{$7 \times 7,64$, stride 2} & & & & & & \\
\hline \multirow[b]{2}{*}{ Conv_2 } & \multirow[b]{2}{*}{$56 \times 56$} & \multicolumn{2}{|c|}{$3 \times 3$, max pool, stride 2} & & & & & & \\
\hline & & {$\left[\begin{array}{lll}3 & x & 3,64 \\
3 & x & 3,64\end{array}\right] x 2$} & {$\left[\begin{array}{lll}3 & x & 3,64 \\
3 & x & 3,64\end{array}\right] x 3$} & $\left|\begin{array}{ccc}1 & x & 1,64 \\
3 & x & 3,64 \\
1 & x & 1,256\end{array}\right|$ & $x 3$ & $\left.\mid \begin{array}{ccc}1 & x & 1,64 \\
3 & x & 3,64 \\
1 & x & 1,256\end{array}\right\rfloor$ & $x 3$ & {$\left[\begin{array}{ccc}1 & x & 1,64 \\
3 & x & 3,64 \\
1 & x & 1,256\end{array}\right]$} & $x 3$ \\
\hline Conv_3 & $28 \times 28$ & {$\left[\begin{array}{lll}3 & x & 3,128 \\
3 & x & 3,128\end{array}\right] x 2$} & {$\left[\begin{array}{lll}3 & x & 3,128 \\
3 & x & 3,128\end{array}\right] x 4$} & {$\left[\begin{array}{lll}1 & x & 1,128 \\
3 & x & 3,128 \\
1 & x & 1,512\end{array}\right]$} & $x 4$ & {$\left[\begin{array}{ccc}1 & x & 1,128 \\
3 & x & 3,128 \\
1 & x & 1,512\end{array}\right]$} & $x 4$ & $\left\lfloor\begin{array}{ccc}1 & x & 1,128 \\
3 & x & 3,128 \\
1 & x & 1,512\end{array}\right\rfloor$ & $x 4$ \\
\hline Conv_4 & $14 \times 14$ & {$\left[\begin{array}{lll}3 & x & 3,256 \\
3 & x & 3,256\end{array}\right] x 2$} & {$\left[\begin{array}{lll}3 & x & 3,256 \\
3 & x & 3,256\end{array}\right] x 6$} & 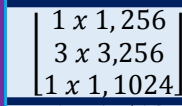 & $x 6$ & {$\left[\begin{array}{ccc}1 & x & 1,256 \\
3 & x & 3,256 \\
1 & x & 1,1024\end{array}\right]$} & $x 23$ & 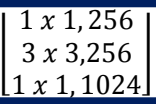 & $x 36$ \\
\hline \multirow[t]{2}{*}{ Conv_5 } & $7 \times 7$ & {$\left[\begin{array}{lll}3 & x & 3,512 \\
3 & x & 3,512\end{array}\right] x 2$} & {$\left[\begin{array}{lll}3 & x & 3,512 \\
3 & x & 3,512\end{array}\right] x 3$} & {$\left[\begin{array}{ccc}1 & x & 1,512 \\
3 & x & 3,512 \\
1 & x & 1,2048\end{array}\right]$} & $x 3$ & $\mid \begin{array}{c}1 \times 1,512 \\
3 \times 3,512 \\
1 \times x\end{array}$ & $x 3$ & 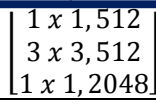 & $x 3$ \\
\hline & $1 \times 1$ & & & \multirow{2}{*}{\multicolumn{2}{|c|}{$3.8 \times 10^{9}$}} & \multicolumn{2}{|l|}{ fc, softmax } & & \\
\hline \multicolumn{2}{|c|}{ FLOPS } & $1.8 \times 10^{9}$ & $3.6 \times 10^{9}$ & & & $7.6 \times 10^{9}$ & & \multicolumn{2}{|l|}{$11.3 \times 10^{9}$} \\
\hline
\end{tabular}

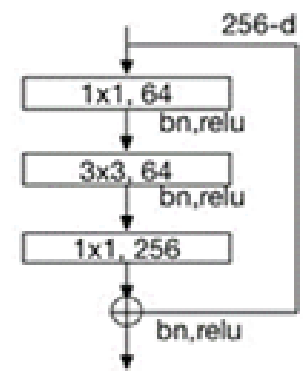

Fig. 3 - The Bottleneck building block.

\subsubsection{Inception-V3 Architecture}

Inception model has few versions such as Inception V1 known as GoogleLeNet, Inception-V2, Inception-V3, Inception-V4 and Inception Resnet-V2 by (Szegedy et al., 2016). Inception-1 architecture is also known as GoogLeNet developed based on LeNet architecture by adding inception module. In this study, we used Inception-V3 architecture by (Szegedy, Vanhoucke, Ioffe, Shlens, \& Wojna, 2015) as shown in Figure 4 to train and test our IVUS images to detect either calcification absent or calcification present . 


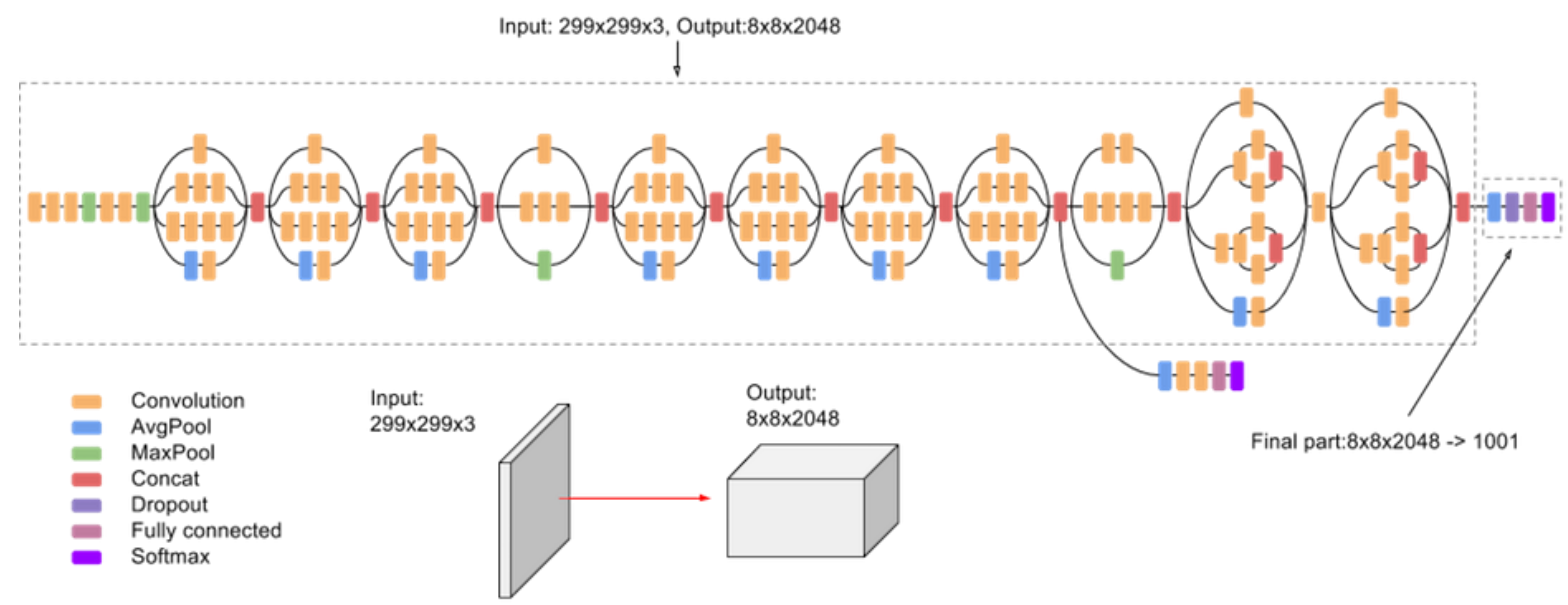

Fig. 4 - The Inception-V3 architecture

\subsection{Experimental Setup}

The proposed framework is as Fig. 5. During the pre-processing step, the original image was resized to $224 \times 224 \times 3-$ channels for ResNet-50 and ResNet-101 while for inception-V3 will resize to 299x299x3 channels. In this step, the Cartesian coordinate image as shown in Figure 5a and Figure $5 \mathrm{~b}$ will be resized based on the CNNs architecture input requirement. Next, the image will be converted into RGB (3-channels). The image is then ready for deep structured learning. The images will be passed through the CNN architecture such as ResNet-50. The output for ResNet-50 is the fully connected layer, fc1000. In the next step, after going through with the deep features training using selected fully connected, fc layers that is fc1000, we normalised the result to get the output in positive values only. The next step is the classification validation, which was training and testing. By using $\mathrm{k}$-fold, the dataset $\mathrm{B}$ was divided into two parts that were testing and training. We classify using three types classifiers which are support decision tree classifier, $\mathrm{k}$-nearest neighbour classifier and naive Bayesian classifier, with $\mathrm{k}$-fold for training and testing (where $\mathrm{k}=2$ ). The $\mathrm{k}$-fold is defined as Testing $=1 / \mathrm{k}$, Training $=(\mathrm{k}-1) / \mathrm{k}$. The train classifier dataset was assigned with labels, ' 1 ' and ' 0 ' where ' 1 ' for calcification present and ' 0 ' for calcification absent. For the final step, the verification of the results done by comparing it with the ground truth provided by the MICCAI Challenge 2011 where the accuracy (Acc), the specificity (Sp), the sensitivity (Sn), the positive predictive value (PPV) and the negative predictive value (NPV) were obtained.

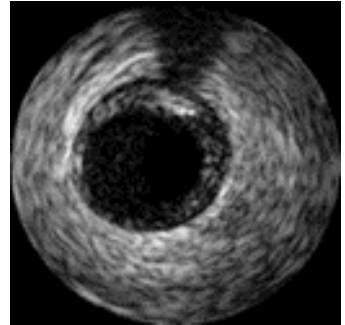

(a)

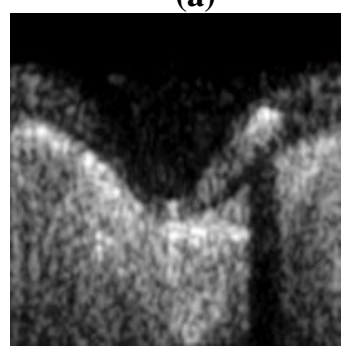

(b)

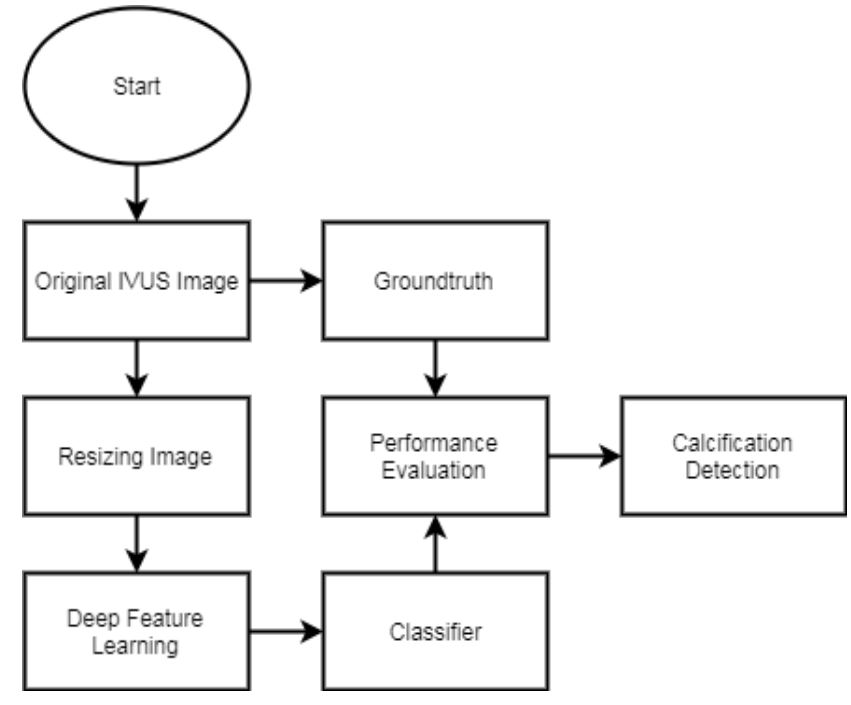

(c)

Fig. 5 - (a) Sample image of Cartesian coordinate (b) Sample image of reconstructed polar coordinate (c) The Calcification Detection Framework. 


\subsection{Performance Measures}

In Table 2 shows the four outcomes from the Confusion Matrix that are the True Positive (TP), the True Negative (TN), the False Positive (FP) and the False Negative (FN). The true positive (TP) is the system was able to categorise the artery disease with calcification present. The false positive (FP) is the artery disease had a calcification absent but recognised as calcification present. The true negative, (TN) is the system was able to categorise the artery disease with calcification absent. The false negative, $(\mathrm{FN})$ is the artery disease had calcification present but detected as calcification absent.

Table 2 - Confusion Matrix

\begin{tabular}{|l|c|c|}
\hline & \multicolumn{2}{|c|}{ Actual Class } \\
\hline Predicted Class & Calcification Present & Calcification Absent \\
\hline Calcification Present & True Positive, TP & True Negative, TN \\
\hline Calcification Absent & False Negative, FP & False Negative, FN \\
\hline
\end{tabular}

The Table 3 shows the Accuracy (Acc), Sensitivity (Sn), Specificity (Sp), Positive Predictive Value (PPV) and Negative Predictive Value (NPV) by (Sofian et al. 2017). The Accuracy (ACC) is defined as the true positive (TP) and the true negative $(\mathrm{TN})$ then divided by the total number of the images. The value one is to indicate the highest accuracy rate, and zero is the worst rate. The sensitivity is the true positive divided by all the correct positive prediction. The specificity is true negative divided by all the correct negative prediction. The positive predictive value (PPV) is defined as the correct prediction positive divided by the total number of positive prediction, and the negative predictive value (NPV) is defined as the correct predictive negative divided by the total number of negative prediction.

Table 3 - The Performance Measures

\begin{tabular}{|l|l|}
\hline Accuracy (Acc) & $(\mathrm{TP}+\mathrm{TN}) /(\mathrm{TP}+\mathrm{TN}+\mathrm{FP}+\mathrm{FN})$ \\
\hline Sensitivity $(\mathrm{Sn})$ & $\mathrm{TP} /(\mathrm{TP}+\mathrm{FN})$ \\
\hline Specificity $(\mathrm{Sp})$ & $\mathrm{TN} /(\mathrm{TN}+\mathrm{FP})$ \\
\hline Positive Predictive Value (PPV) & $\mathrm{TP} /(\mathrm{TP}+\mathrm{FP})$ \\
\hline Negative Predictive Value (NPV) & $\mathrm{TN} /(\mathrm{TN}+\mathrm{FN})$ \\
\hline
\end{tabular}

\subsection{Validation}

For the validation, we used k-fold cross-validation technique to access the performance measures results of the CNNs models to the independent datasets. The value of $\mathrm{k}$-fold cross-validation taken is 2, 3, 5 and 10 . The $\mathrm{k}$-fold is defined as:

$$
\text { Training }=\frac{k-1}{k} ; \quad \text { Testing }=\frac{1}{k}
$$

The train classifier dataset was assigned with labels, ' 1 ' and ' 0 ' where ' 1 ' for calcification present and ' 0 ' for calcification absent. The datasets will be divided into two parts, cross-validation training data for the known data and cross-validation testing data for the unknown data. Therefore, to get the accuracy performance measures results, the CNNs model's prediction results will be compared between the CNNs model prediction validation set and the actual labels of the validation sets. For all the iterations, the average results will be taken for the accuracy estimation for the CNNs model.

\section{Result and Discussion}

In our experiments, the image input used were two types of images, the Cartesian coordinate image and the polar reconstructed coordinate image. Each of the images will be fed into the ResNet-50 architecture with the output layer taken at fc1000 layer then. The same method also used for the ResNet-101 architecture and Inception-V3. We used five types of classifiers to find the best performance measures result that are Support Vector Machine, Discriminant Analysis, Decision Tree, K- Nearest Neighbours and Naive Bayes. For validation, we used k-fold cross-validation with 2-fold, 3fold, 5-fold and 10-fold for the testing and training. The performance measures were the accuracy, sensitivity, specificity, positive predictive value and negative predictive value. The results performance measures were compared to the MICCAI 2011 Challenged ground truth. 


\subsection{Elapse Time}

The Table 4 shows the elapsed time comparison between the two types of images (Cartesian coordinate image and polar reconstructed image) using three types of CNNs models (ResNet-50, ResNet-101 and Inception-V3). The first column was a ResNet-50 model; the second column was a Resnet-101 model and the third column was an Inception-V3 model. The first row of Cartesian coordinate (CC) image shows that the time taken for model ResNet-50 took less time compared to ResNet-101 followed by Inception-V3. After the Cartesian coordinate image shifted to polar reconstructed coordinate (PRC) image, ResNet-50 (the blue bar) took less time compared to ResNet-101 (the orange bar) followed by Inception-V3 (the grey bar). Overall, both images from ResNet-50 model shows the fastest elapsed time, 8453 seconds for Cartesian coordinate image and 12374.94 seconds for polar reconstructed coordinate (PRC) image taken for training feature when compared to ResNet-101 and Inception-V3 as shown in Figure 9. The longest elapse time taken was Inception-V3 using original image (CC Image), 50133.23 seconds.

Table 4 - Training Features Elapse Time

\begin{tabular}{|l|c|c|c|}
\hline \multicolumn{3}{|c|}{ Training Features Elapse Time in seconds } \\
\hline Images & ResNet-50 & ResNet-101 & 16540.39 \\
\hline Cartesian Coordinate Image & 8453 & 50133.23 & 16429.33 \\
\hline Polar Reconstructed Coordinate Image & 12374.94 & 12600.07 & \\
\hline
\end{tabular}

\subsection{Calcification Detection}

The result of performance measures is as shown in Figure 7, Figure 8, Figure 9, Figure 10 and Figure 11. The two type of images, Cartesian coordinate image (CC) and polar reconstructed coordinate image (PRC) were compared with the five types of classifiers; decision tree (DT), K-Nearest Neighbour (K-NN), Naïve Bayes (NB) support vector machine classifier and discriminant analysis classifier fed to the three types of convolutional neural networks (CNNs) architectures; ResNet-50 (RN-50), ResNet-101(RN-101) and Inception-V3(I-V3). For the validation, the training and testing for $\mathrm{k}$-fold cross-validation results were taken value $\mathrm{K}=2,3,5$ and 10 . The performance measures show results shows that support vector machine classifier and discriminant analysis classifier show an excellent result when applying to both types of images and all types of convolutional neural networks (CNNs) architectures.

In Figure 7 of accuracy shows the decision tree classifiers, indicates in blue colour, k-nearest neighbour indicates in green colour, naïve Bayes indicates in orange colour, support vector machine indicates grey colour and discriminant analysis classifier indicates yellow colour. For 2-fold, Cartesian coordinate image applying to the Inception - V3 architecture with naïve Bayes shows the highest result with value 0.9917 compared to a polar reconstructed coordinate image with value 0.9770 . For 3 -fold, the polar reconstructed coordinate image applying to the ResNet-101 architecture with k-nearest neighbour shows the highest value with 9931 compared to Cartesian coordinate image 0.9585. For 5-fold polar reconstructed coordinate images applying to the ResNet-101 architecture with k-nearest neighbour shows the highest value with 9954 compared to Cartesian coordinate image 0.9697 . For 10 -fold, the polar reconstructed coordinate image applying to the ResNet-101 architecture with k-nearest neighbour shows the highest value with 1 compared to Cartesian coordinate image 0.9862. Therefore, it shows that the best accuracy is when applying 10-fold using polar reconstructed coordinate image using classifier k-nearest neighbour and ResNet-101 architectures. For 10-fold, decision tree classifier shows the excellence performance measures using all both images when applying all classification compared to another two classifiers.

The sensitivity in Figure 8 shows the decision tree classifier, indicates in blue colour, k-nearest neighbour indicates in orange colour, naïve Bayes indicates in grey colour, support vector machine indicates yellow colour and discriminant analysis classifier indicates red colour. For 2-fold, Cartesian coordinate image applying to the inception - V3 architecture with naïve Bayes shows the highest result with value 1 compared to a polar reconstructed coordinate image with value 0.9717. For 3-fold, polar reconstructed coordinate image applying to the ResNet-50 architecture using K-nearest neighbour classifier and Inception-V3 architecture using decision tree classifier, Cartesian coordinate image applying to the ResNet-101 architecture using Naïve Bayes classifier and inception-V3 using decision tree classifier shows the highest value with 1. For 5-fold with value 1, Cartesian coordinate images using naïve Bayes for Cartesian coordinate applying to ResNet101 and InceptionV3 architectures as well as polar reconstructed coordinate image, ResNet-101 using K-nearest neighbour architecture and Inception-V3 architecture using naïve Bayes. For 10-fold, the polar reconstructed coordinate image shows the best result compared to the Cartesian coordinate image. Inception-V3 architecture shows the value 1 for all classifiers. ResNet-50 shows value 1 for Decision tree classifier using Cartesian coordinate image whilst Naïve Bayes and K-nearest neighbour classifiers using the polar reconstructed coordinate image. ResNet-101 shows value 1 for the Cartesian coordinate image using naïve Bayes and polar reconstructed coordinate image using k-nearest neighbour classifier. 


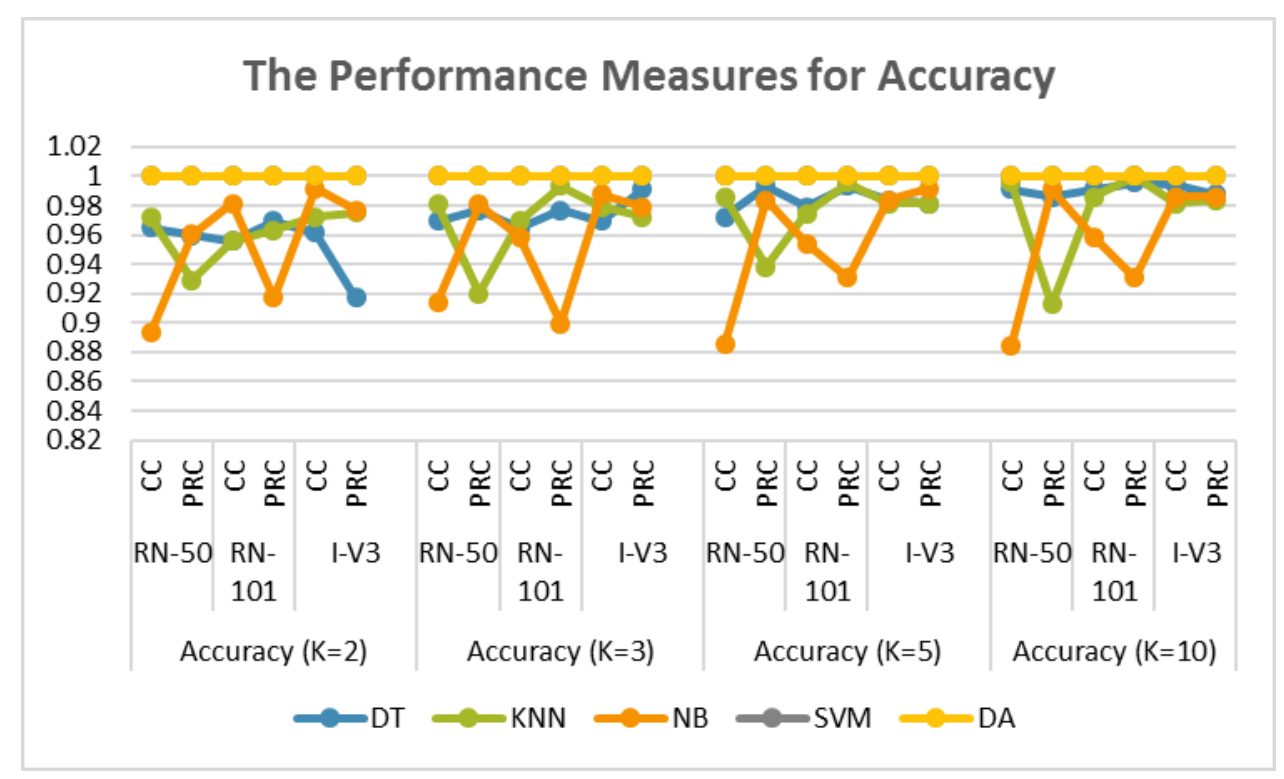

Fig. 7 - The Performance Measure for Accuracy.

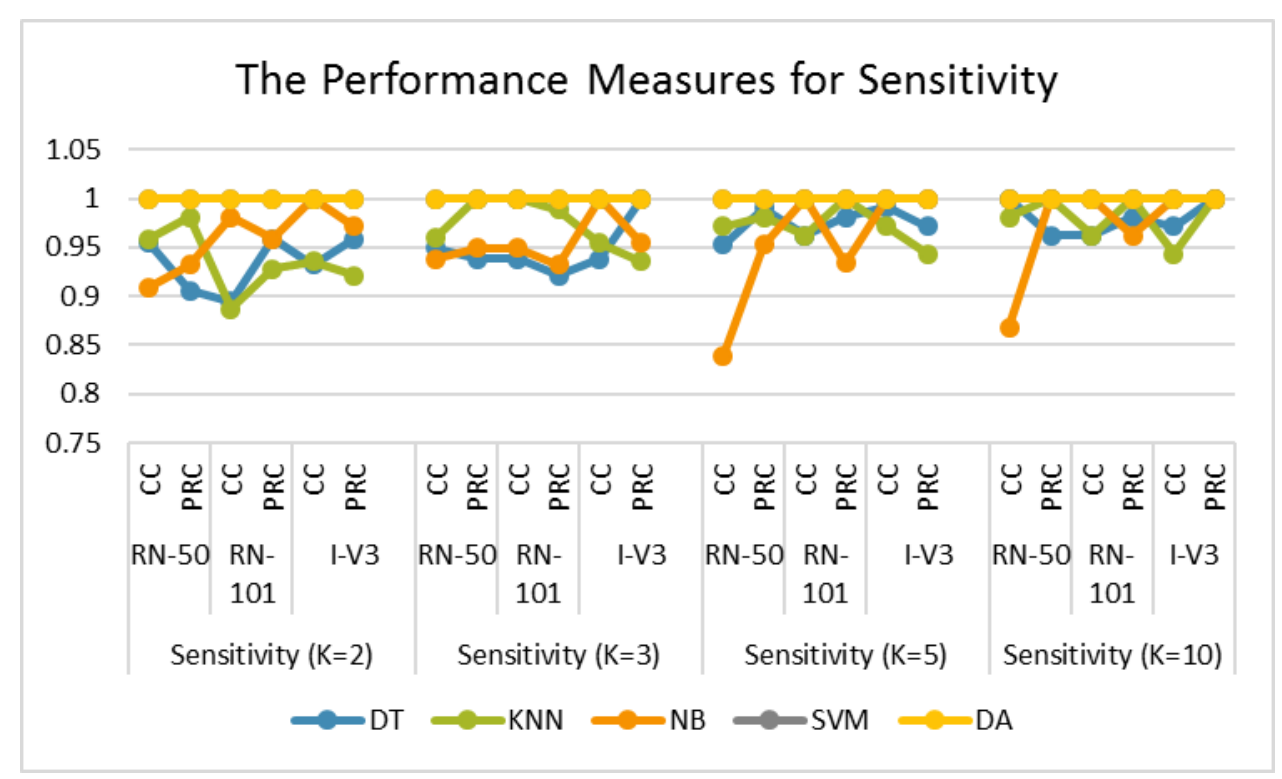

Fig. 8 - The Performance Measure for Sensitivity.

In Figure 9 shows the specificity results. For the decision tree classifiers, indicates in blue colour, k-nearest neighbour indicates in green colour, naïve Bayes indicates in orange colour, support vector machine indicates grey colour and discriminant analysis classifier indicates yellow colour. For 2-fold, Cartesian coordinate image applying to the inception - V3 architecture with naïve Bayes shows the highest result with value 1 compared to a polar reconstructed coordinate image with value 0.9717 . For 3 -fold, the Cartesian coordinate image shows the highest value 1 for the ResNet101 architecture using k-nearest neighbour classifier and Inception-V3 using naïve Bayes classifier and also for the polar reconstructed coordinate image when applying ResNet-50 architecture using k-nearest neighbour and Inception-V3 architecture using Decision tree classifier.

The positive predictive value in Figure 10 shows the results almost near to each other. The decision tree classifiers, indicates in baby blue colour, k-nearest neighbour indicates in orange colour, naïve Bayes indicates in grey colour, support vector machine indicates yellow colour and discriminant analysis classifier indicates dark blue colour. For 2fold, the polar reconstructed coordinate image highest value 0.9760 compared to Cartesian coordinate image value 0.9502 coordinate images when using naïve Bayes and polar reconstructed coordinate image using k-nearest neighbour classifier. For 3-fold, decision tree and k-nearest neighbour classifiers show the highest result when using a polar reconstructed coordinate image when applying to ResNet-101 architecture with value 0.9945 . For 5-fold, the highest value is 0.997 
when applying to the ResNet-101 architecture using the polar reconstructed image and k-nearest neighbour classifier compared to a Cartesian coordinate image with value 0.9533 . For 10 -fold, the highest value 1 when applying polar reconstructed coordinate image in ResNet-101 architecture using decision tree and k-nearest neighbour classifiers whilst Cartesian coordinate image when using a Res-50 architecture with k-nearest neighbour classification and ResNet-101 with decision tree classifier.

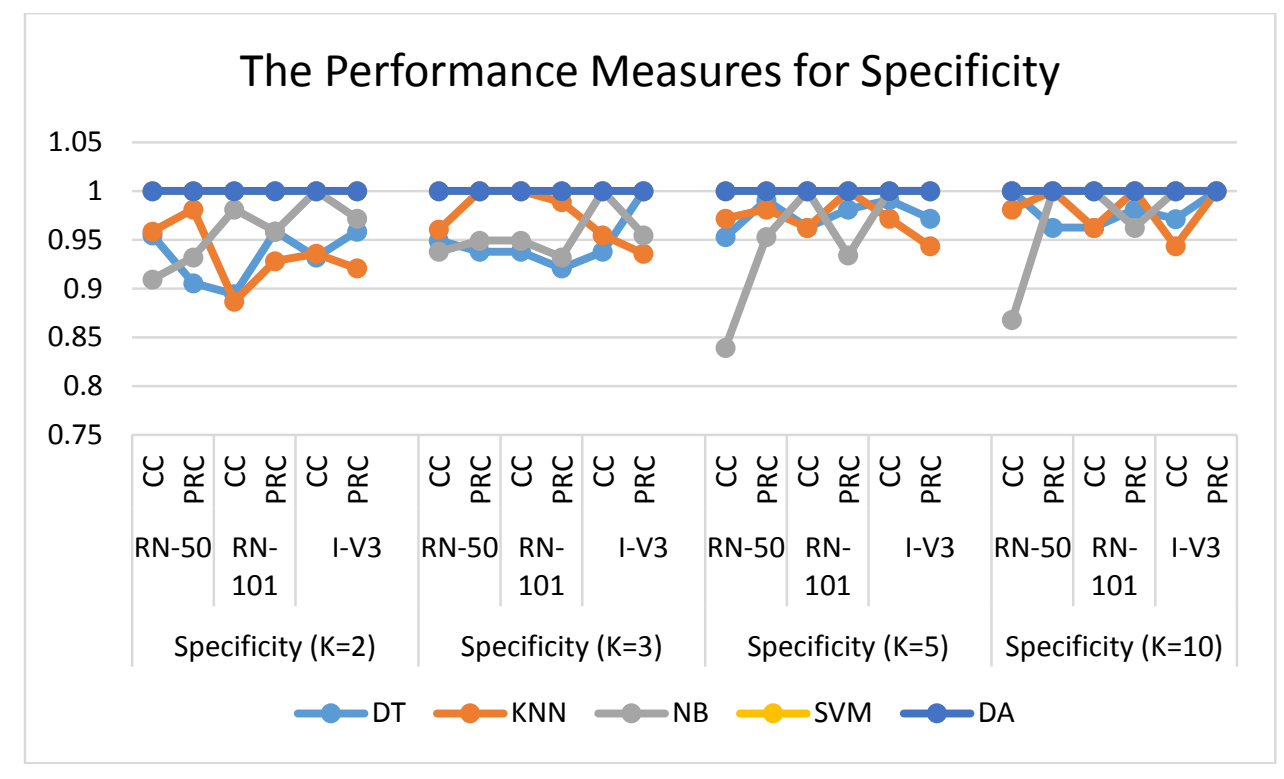

Fig. 9 - The Performance Measure for Specificity.

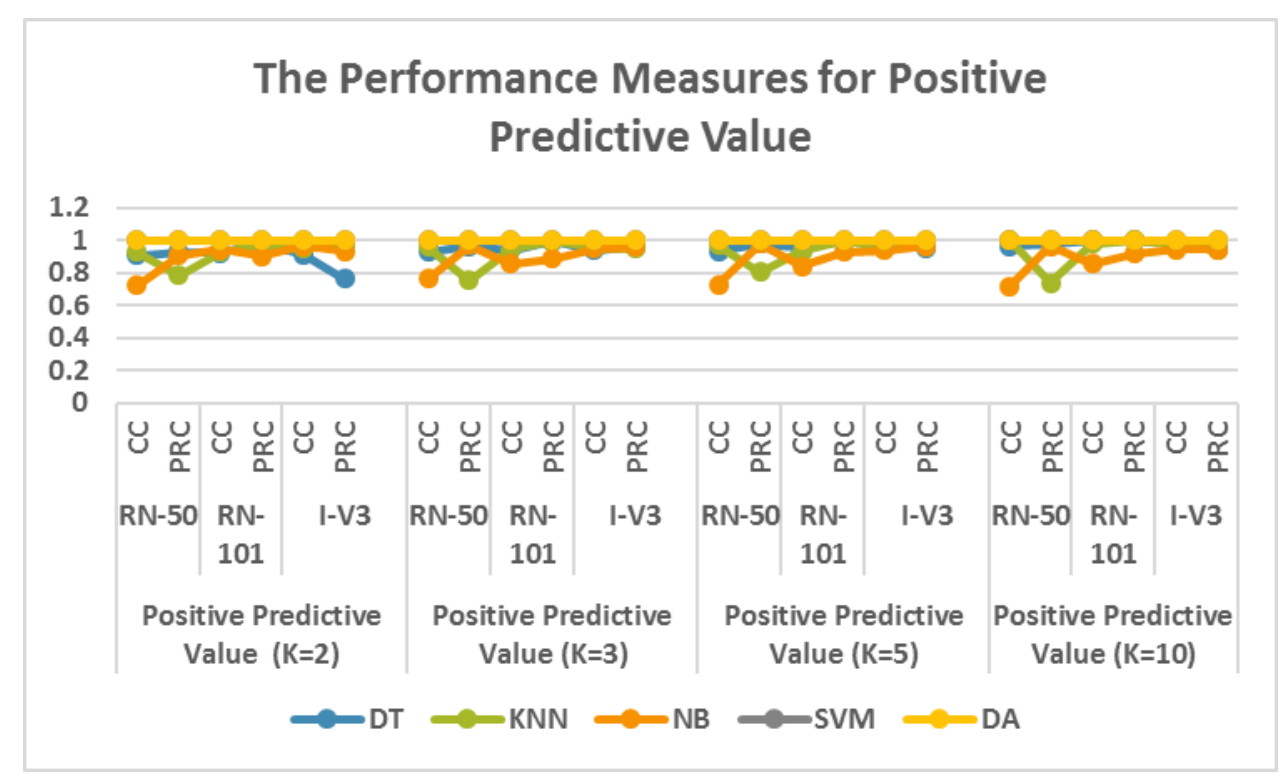

Fig. 10 - The Performance Measure for Positive Prediction Value.

Figure 11 shows the results for negative predictive value were quite scattered compared to positive predictive value. The negative predictive value shows the decision tree classifiers, indicates in blue colour, k-nearest neighbour indicates in green colour, naïve Bayes indicates in orange colour, support vector machine indicates grey colour and discriminant analysis classifier indicates yellow colour. For 2-fold, Cartesian coordinate images show the highest value 1 compared to a polar reconstructed coordinate image with value 0.9908 when applying to inception-V3 architecture. For 3-fold, Inception-V3 shows the highest value for both images using naïve Bayes for Cartesian coordinate and decision tree for polar reconstructed coordinate image whilst ResNet-50 when using k-nearest neighbour with polar reconstructed coordinate image and ResNet-101 when using Cartesian coordinate image using naïve Bayes. For k-5, naïve Bayes shows 
the highest value 1 when applying to Cartesian coordinate image and polar reconstructed coordinate image in inceptionV3 architecture. For ResNet-101 architecture, value 1 shows in k-nearest neighbours for polar reconstructed coordinate image and naïve Bayes in the Cartesian coordinate image. For 10-fold same as 5-fold, however, the highest results had shown an improvement in ResNet-50 architecture for the Cartesian coordinate image when using decision tree classifier.

\section{The Performance Measures for Negative Predictive Value}

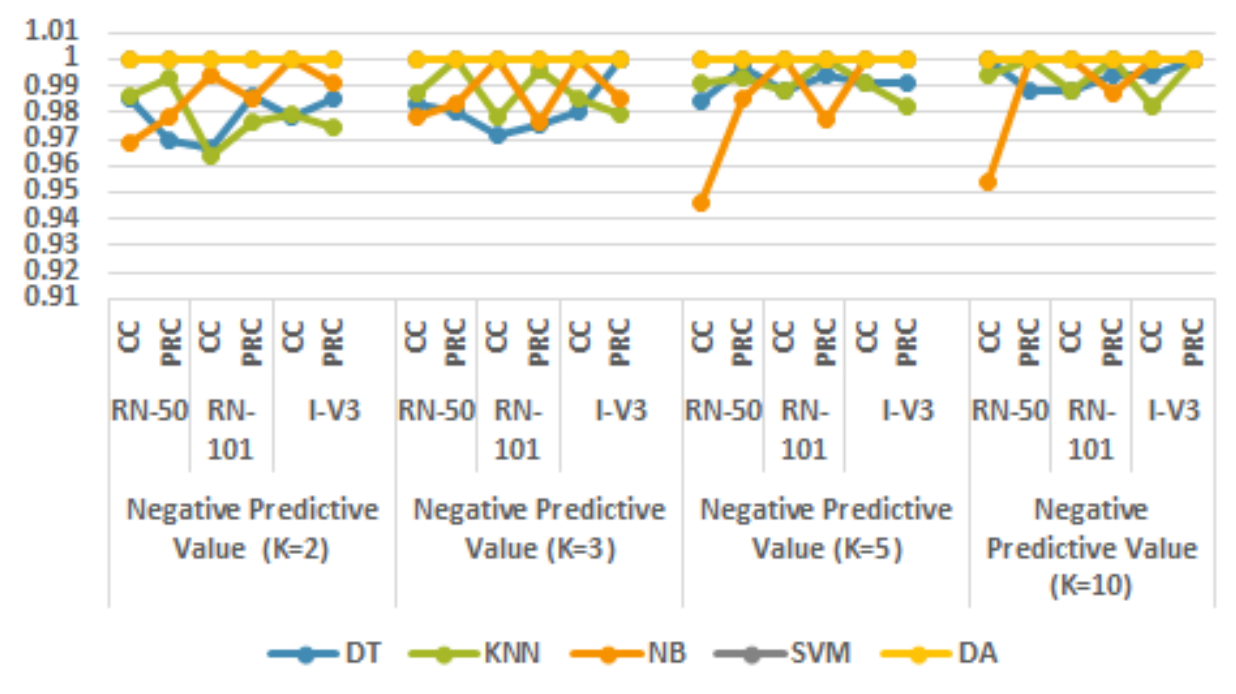

Fig. 11 - The Performance Measure for Negative Prediction Value.

\subsection{Results of the CNNs Models}

The Receiver Operating Characteristics (ROC) curve used to predict the accuracy of the performance measures by (Fawcett, 2006). The receiver operating characteristics curve results of the calcification detection were shown in Figure 10, Figure 11, Figure 12 and Figure 13, respectively. The sensitivity versus 1 -specificity were shown as the y-axis is referred to the sensitivity while $\mathrm{x}$-axis is referring to 1-specificity. The graph indicates the Decision Tree classifier (Dark Blue line), K-Nearest Neighbour classifier (Green line), naïve Bayes classifier (Cyan line), Support Vector Machine (Yellow line) and Discriminant Analysis classifier (red line). Overall, the graph shows that the curve is near to the y-axis and close to the top of the x-axis. Moreover, the closer curve to the $\mathrm{y}$-axis and top of the $\mathrm{x}$-axis shows the accuracy of the calcification detection. All the curves are above than the 45 degrees line (The black line) of the Receiver Operating Characteristics (ROC) curve. The graph that shows the excellent result is above value 0.9 , between value 0.8 to 0.9 is consider good; the fair results are between value 0.7 to 0.8 , the poor results are between value 0.6 to 0.7 and between value 0.5 to 0.6 is considered fail.

In Figure 12, the ROCs for the cross-validation for 2-fold are shown. All the CNNs models show that the Support Vector Machine classifier and Discriminant Analysis shows the excellent performance measure results that are all value one. Therefore the ROC curve is one. For K-Nearest neighbour classifier, Naïve Bayes classifier and decision tree classifier also show the excellent curve results that are above 0.9 and area above than 0.5 . As a conclusion, the original images known as Cartesian coordinate image and polar reconstructed coordinate images gave excellent results.

In Figure 13, the ROCs for the cross-validation for 3-fold are shown. All the CNNs models show that the Support Vector Machine classifier and Discriminant Analysis shows the excellent results that is 1 for the curve and the area. For K-Nearest neighbour classifier, Naïve Bayes classifier and decision tree classifier also show the excellent curve results that are above 0.9 and area above than 0.5 . The ROC curve results were better when $\mathrm{k}=2$. As a conclusion, the original images known as Cartesian coordinate image and polar reconstructed coordinate images gave excellent results.

In Figure 14 shows the ROCs for the cross-validation for 5-fold. The Support Vector Machine classifier and Discriminant Analysis classifier shows the excellent result that is value one. The ROC curve value obtained is one. For K-Nearest neighbour classifier, Naïve Bayes classifier and decision tree classifier also show the excellent curve results that are above 0.95 and area above than 0.5 . The ROC curve results were better when value $\mathrm{k}-$ fold, $\mathrm{k}=3$. As a conclusion, 
the original images known as Cartesian coordinate image and polar reconstructed coordinate images gave excellent results.

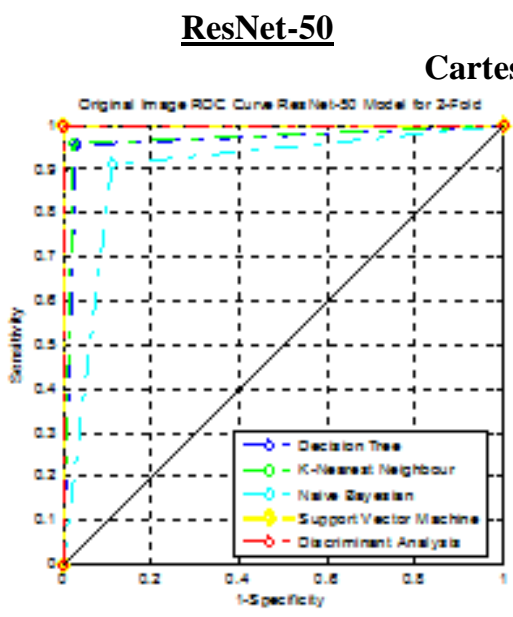

(a)

ResNet-101 Cartesian Coordinate Image (Original Image) , $\mathrm{K}=2$

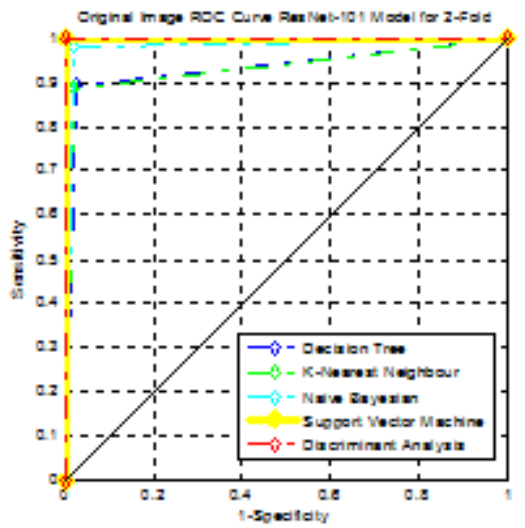

(b)

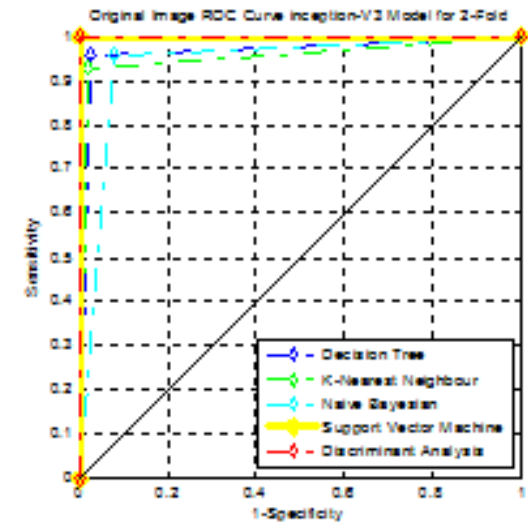

(c)

Polar Reconstructed Coordinate Image, $K=2$

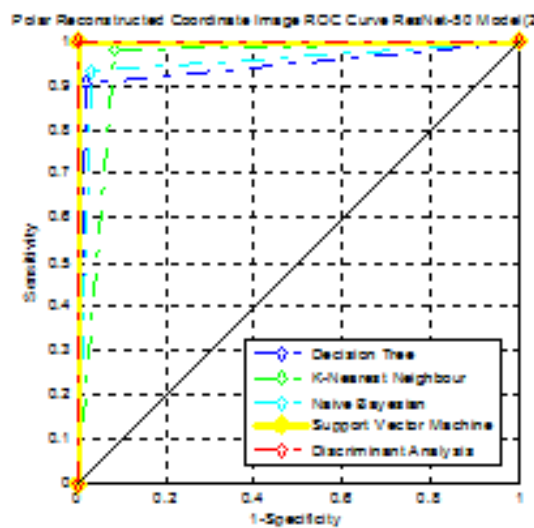

(d)

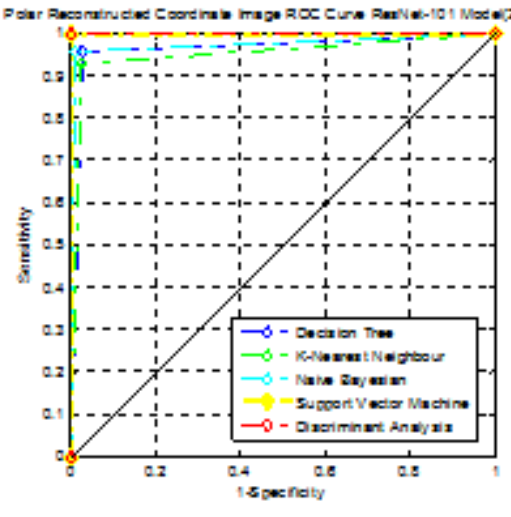

(e)

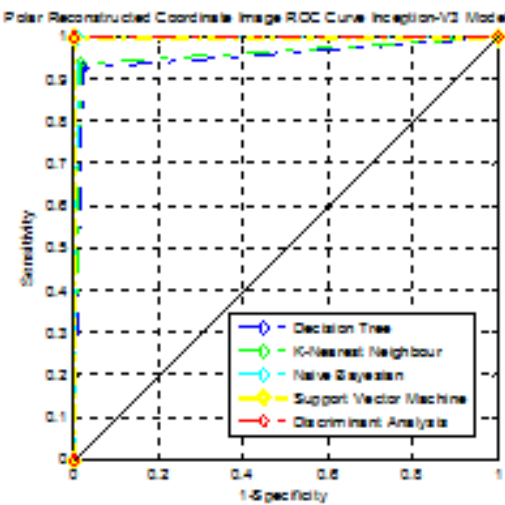

(f)

Fig. 12 - The Receiver Operating Graph (ROC) curve for $\mathrm{K}=2$

$\underline{\text { ResNet-50 }}$

ResNet-101 Cartesian Coordinate Image (Original Image) , $\mathrm{K}=3$

$\underline{\text { Inception-V3 }}$

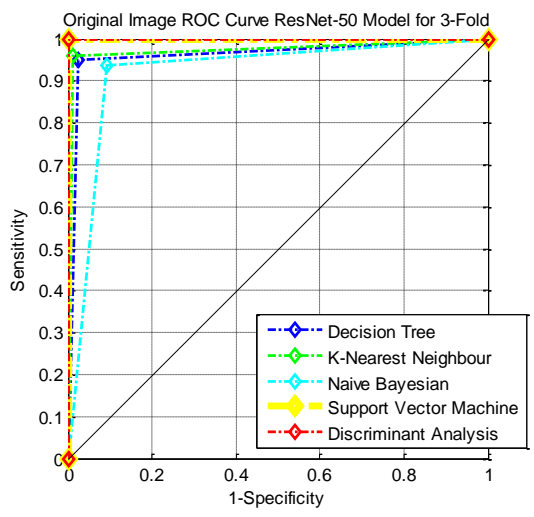

(a)

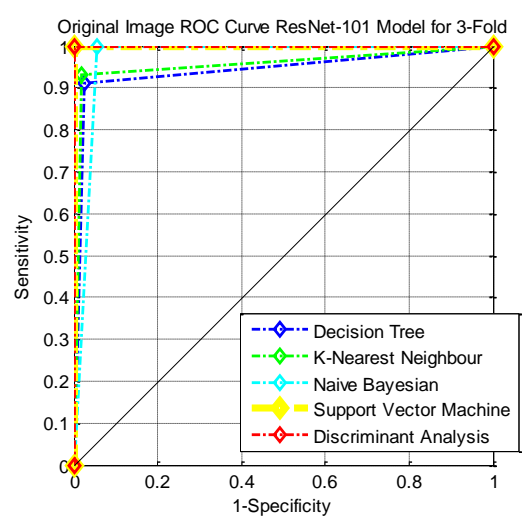

(b)

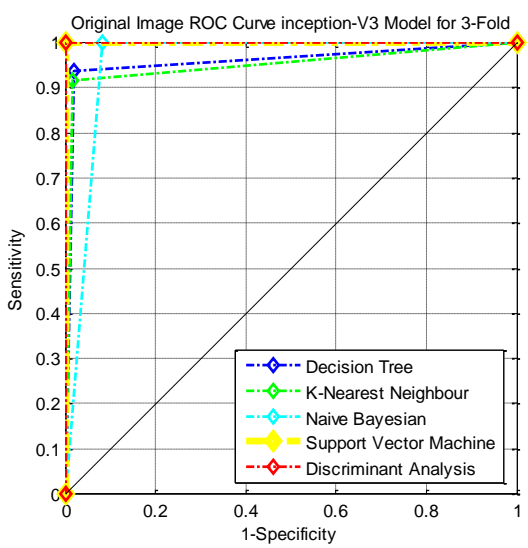

(c) 
Polar Reconstructed Coordinate Image, $\mathrm{K}=3$

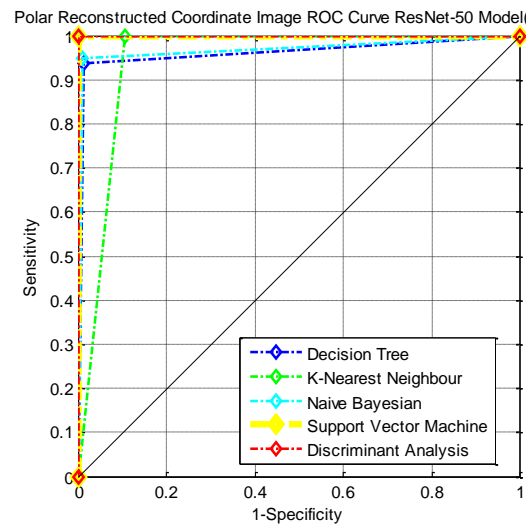

(d)

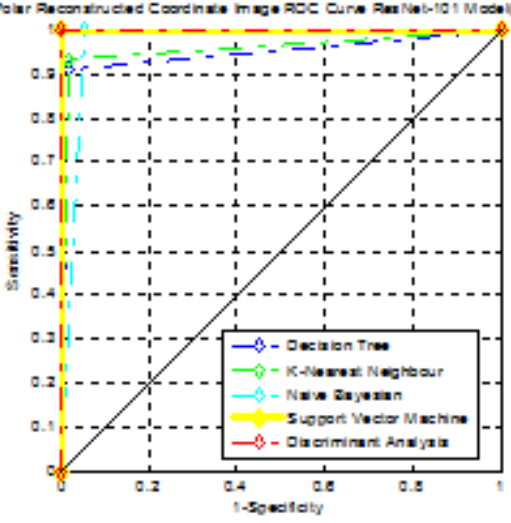

(e)

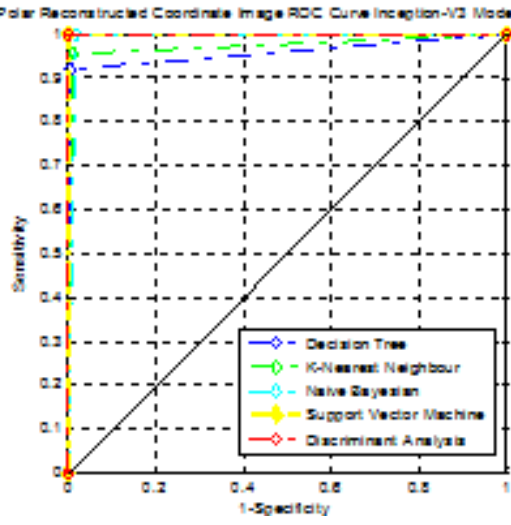

(f)

Fig. 13 - The Receiver Operating Graph (ROC) curve for $\mathrm{K}=3$

\section{$\underline{\text { ResNet-50 }}$}

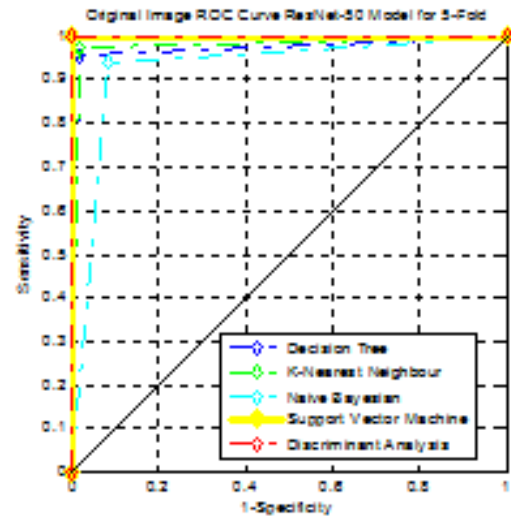

(a)

ResNet,-101 The Cartesian Coordinate Image (Original Image), $K=5$

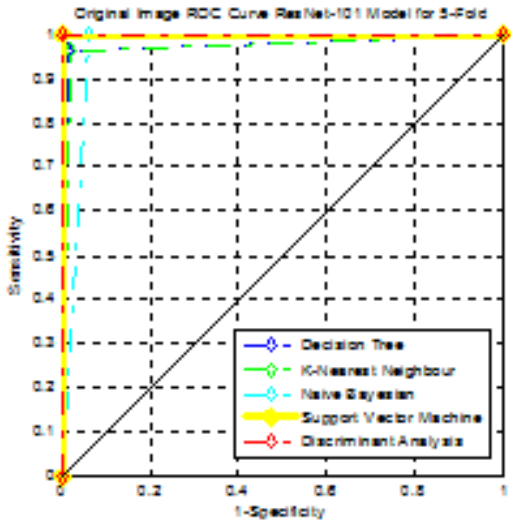

(b)

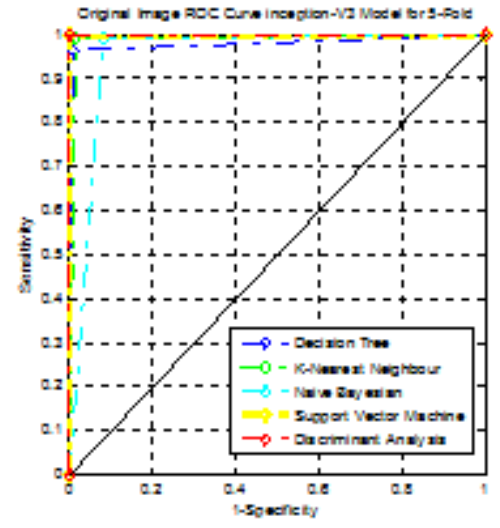

(c)

Polar Reconstructed Coordinate Image, $\mathrm{K}=5$

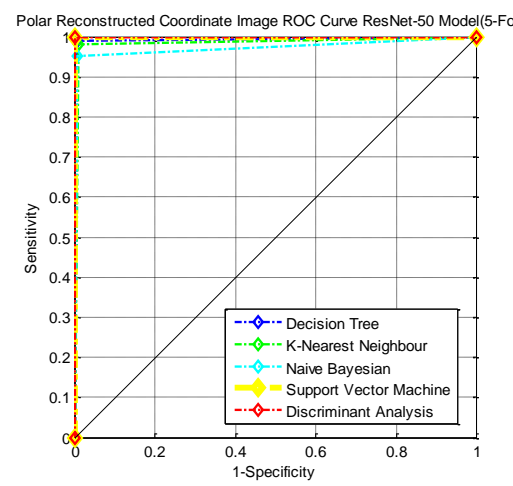

(d)

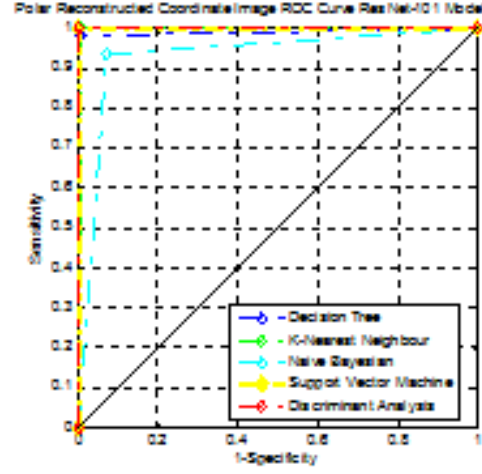

(e)

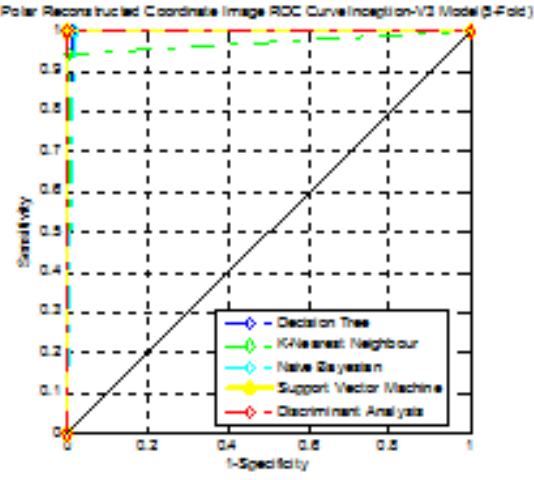

(f)

Fig. 14 - The Receiver Operating Graph (ROC) curve for $K=5$.

In Fig. 15, the ROCs for the cross-validation for 10-fold are shown. All the CNNs models show excellent results. However, the Support Vector Machine classifier and Discriminant Analysis shows the excellent results that is 1 for the ROC curve. For K-Nearest neighbour classifier, Naïve Bayes classifier and decision tree classifier also show the excellent curve results that are above 0.95 and area above than 0.5. Unfortunately, Naïve Bayes classifier in Fig. 13a shows a good result that is above 0.85 . The ROC curve results were better compared to $k=5$; however, for Naïve Bayes using the 
Cartesian coordinate image for ResNet-50 model, the ROC curve is below than 0.9 . As a conclusion, the original images known as Cartesian coordinate image and polar reconstructed coordinate images gave excellent results.

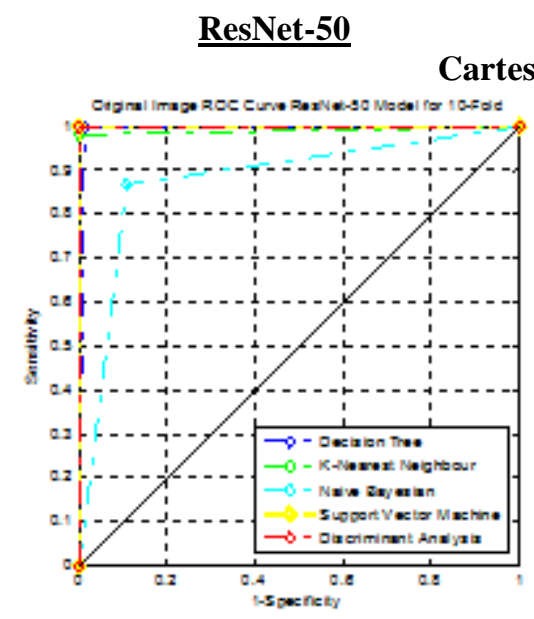

(a)
ResNet-101

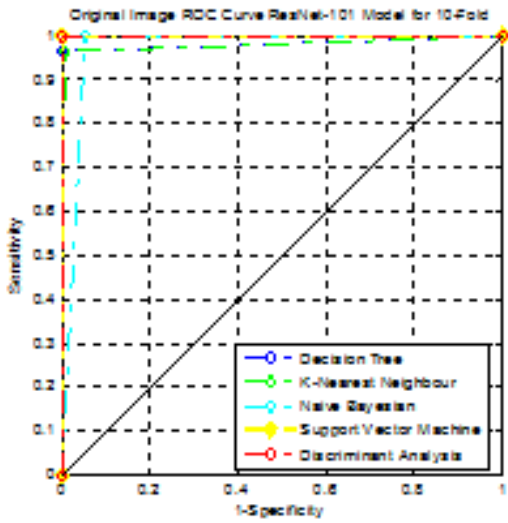

(b) $\underline{\text { Inception-V3 }}$

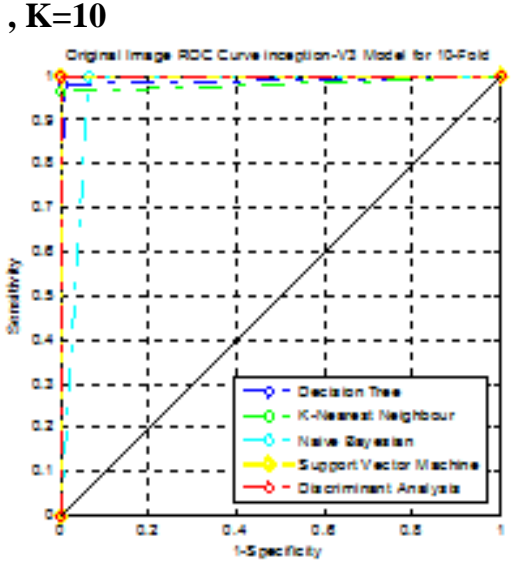

(c)

Polar Reconstructed Coordinate Image, $K=10$

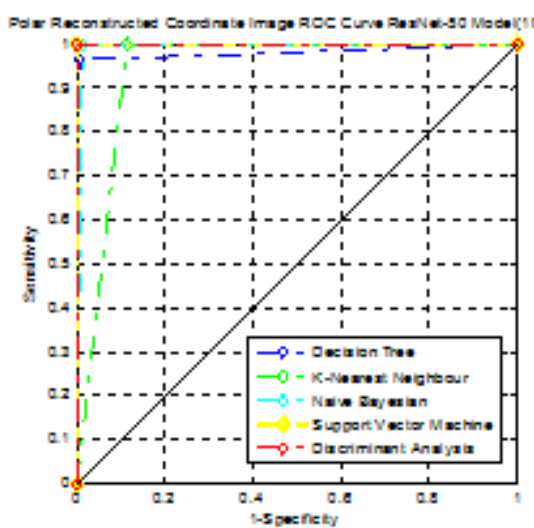

(d)

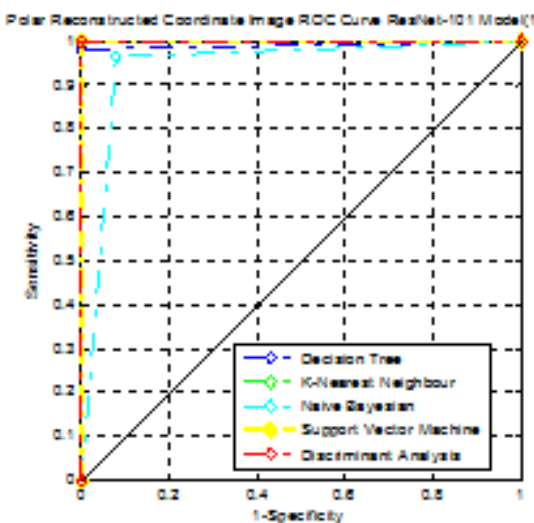

(e)

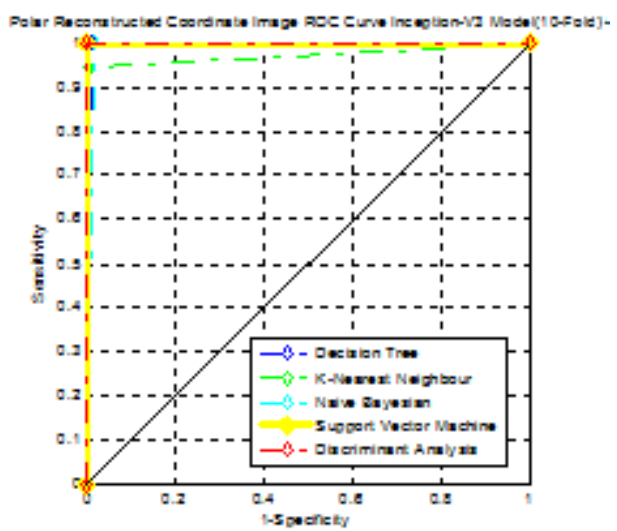

(f)

Fig. 15 - The Receiver Operating Graph (ROC) curve for $\mathrm{K}=10$

Table 5 shows the comparison between the previous methods used to detect the calcification in coronary artery disease using intravascular ultrasound (IVUS) images. By using the deep feature learning for all CNNs models, the accuracy, the sensitivity and the specificity are the highest. The previous researchers that used calcification segmentation to detect the calcification gave a good result that is between 0.83 to 0.98 for sensitivity and 0.81 to 0.96 for specificity (Sofian et al. 2017) used 1645 images without calcification and 530 images with calcification. The method used was Otsu Threshold, morphological operation \& Empirical Threshold and they obtained $82 \%$ accuracy. The methods and technique used by (Gao et al. 2014) obtained 97\% sensitivity and 95\% specificity with 996 images presence calcification and 996 images absence calcification. They used the Rayleigh mixture model, the Markov random field, and the graph searching method to detect calcification. (Taki et al. 2008) used from 60 images using geometric deformable models and Bayesian classifier and obtained $98 \%$ sensitivity and 96\% specificity while (Santos-Filho et al. 2007) used 20 IVUS images using adaptive thresholding to detect calcification.

\section{Conclusion}

This study shows the novelty of the calcification detection whether present or absent calcification by using deep feature learning approach that used two types of images; Cartesian coordinate and polar reconstructed coordinate, three types of CNNs architectures; ResNet-50, ResNet-101 and Inception-V3 and five types of classifiers; support vector machine, discriminant analysis, decision tree, k-nearest neighbours and Naïve Bayes. The receiver operating characteristics (ROC) curve results shown the excellent ROC curve results and accuracy when using support vector machine classifier and discriminant analysis classifier that is value one in both images. By using a reconstructed polar image, K- nearest neighbour classifier and Decision Tree classifier show an excellent with ResNet-101 architecture while 
Naïve Bayes has an excellent result with Inception-V3 architecture. However, the performance measures for the ResNet50, the ResNet-101 and the Inception-V3 model shows an excellent result using support vector machine classifier and discriminant analysis for both types of images. As a conclusion, the polar reconstructed coordinate image shows an excellent result compared to the Cartesian coordinate image.

In future work, work on detecting the plaque and work on the detection of calcification using the different catheter frequency and modality, for example, optical coherence tomography modality including the detection of plaques, bifurcation, Stent Side Vessels and Guidewire Artifacts should be carried out.

Table 5 - The Comparison Performances Measures

\begin{tabular}{|c|c|c|c|c|c|}
\hline $\begin{array}{c}\text { Author, } \\
\text { Year }\end{array}$ & Total Image, Method & $\begin{array}{c}\text { Catheter } \\
\text { Frequency (MHz) }\end{array}$ & Accuracy & Sensitivity & Specificity \\
\hline $\begin{array}{c}\text { Proposed } \\
\text { Method }\end{array}$ & $\begin{array}{c}2175, \text { ResNet-50, ResNet-101 and } \\
\text { Inception-V3 with Support Vector } \\
\text { Machine classifier and } \\
\text { Discriminant Analysis classifier }\end{array}$ & 20 & 1.0000 & 1.0000 & 1.0000 \\
\hline $\begin{array}{c}\text { Sofian et } \\
\text { al.[3] }\end{array}$ & $\begin{array}{c}\text { morphological operation \& } \\
\text { Empirical Threshold }\end{array}$ & 20 & 0.8200 & 0.8300 & 0.8100 \\
\hline $\begin{array}{c}\text { Gao et al. } \\
{[7]}\end{array}$ & $\begin{array}{c}\text { 1992, Rayleigh mixture model, the } \\
\text { Markov random field, and the graph } \\
\text { searching method }\end{array}$ & 20 & NA & 0.9668 & 0.9582 \\
\hline $\begin{array}{c}\text { Taki et al. } \\
{[10]}\end{array}$ & $\begin{array}{c}\text { Random 60, geometric deformable } \\
\text { models and Bayesian classifier }\end{array}$ & 30 & NA & 0.9800 & 0.9300 \\
\hline $\begin{array}{c}\text { Santos-Filho } \\
\text { et al. [16] }\end{array}$ & 20, Adaptive Thresholding & 40 & NA & 0.8400 & 0.8800 \\
\hline
\end{tabular}

Note: NA- Not Available

\section{Acknowledgement}

We would like to thank Prof Simone Balocco, Computer Vision Center, Dept. Matemàtica Aplicada i Anàlisi, Bellaterra-Universitat de Barcelona, Barcelona for his cooperation in this study. The research funded by the Ministry of Higher Education Malaysia, Universiti Teknologi Malaysia Research Grant (Q.K. 130000.2540.14H22) and Universiti Kuala Lumpur.

\section{References}

[1] Balocco, S., Gatta, C., Ciompi, F., Wahle, A., Radeva, P., Carlier, S., ... Kakadiaris, I. a. (2014). Standardized evaluation methodology and reference database for evaluating IVUS image segmentation. Computerized Medical Imaging and Graphics: The Official Journal of the Computerized Medical Imaging Society, 38(2), 70-90. http://doi.org/10.1016/j.compmedimag.2013.07.001

[2] Bdolmanafi, A. T. A., Uong, L. U. C. D., Ahdah, N. A. D., Brahim, I., Dib, R. A. A., \& Heriet, F. A. C. (2018). Characterization of coronary artery pathological formations from OCT imaging using deep learning, 9(10), 7-10.

[3] Bortsova, G., van Tulder, G., Dubost, F., Peng, T., Navab, N., van der Lugt, A., ... De Bruijne, M. (2017). Segmentation of intracranial arterial calcification with deeply supervised residual dropout networks. Lecture Notes in Computer Science (Including Subseries Lecture Notes in Artificial Intelligence and Lecture Notes in Bioinformatics), 10435 LNCS, 356-364. http://doi.org/10.1007/978-3-319-66179-7_41

[4] Daugman, J. G. (1993). High Confidence Visual Recognition of Persons by a Test of Statistical Independence. IEEE Transactions on Pattern Analysis and Machine Intelligence. http://doi.org/10.1109/34.244676

[5] Fawcett, T. (2006). An introduction to ROC analysis. Pattern Recognition Letters, 27(8), 861-874. http://doi.org/10.1016/j.patrec.2005.10.010

[6] Filho, E. S., Saijo, Y., Tanaka, A., Yambe, T., Li, S., \& Yoshizawa, M. (2007). Automated Calcification Detection and Quantification in Intravascular Ultrasound Images by Adaptive Thresholding. IFMBE Proceedings, 14(3), 1421-1425.

[7] Gao, Z., Hau, W. K., Lu, M., Huang, W., Zhang, H., Wu, W., ... Zhang, Y.-T. (2015). Automated Framework for Detecting Lumen and Media-Adventitia Borders in Intravascular Ultrasound Images. Ultrasound in Medicine \& Biology, 41(7), 2001-21. http://doi.org/10.1016/j.ultrasmedbio.2015.03.022

[8] Gessert, N., Lutz, M., Heyder, M., Leistner, S. L. D. M., Abdelwahed, Y. S., \& Schlaefer, A. (2018). Automatic Plaque Detection in IVOCT Pullbacks Using Convolutional Neural Networks, 1-9. http://doi.org/10.1109/TMI.2018.2865659 
[9] He, K., Zhang, X., Ren, S., \& Sun, J. (2015). Deep Residual Learning for Image Recognition. http://doi.org/10.3389/fpsyg.2013.00124

[10] Jodas, D. S., Pereira, A. S., \& Tavares, J. M. R. S. (2017). Automatic segmentation of the lumen region in intravascular images of the coronary artery. Medical Image Analysis, 40(November), 60-79. http://doi.org/10.1016/j.media.2017.06.006

[11] Katouzian, A., Karamalis, A., Sheet, D., Konofagou, E., Baseri, B., Carlier, S. G., ... Laine, A. F. (2012). Iterative self-organizing atherosclerotic tissue labeling in intravascular ultrasound images and comparison with virtual histology. IEEE Transactions on Bio-Medical Engineering, 59(11), 3039-49. http://doi.org/10.1109/TBME.2012.2213338

[12] Krizhevsky, A., Sutskever, I., \& Hinton, G. E. (2012). ImageNet Classification with Deep Convolutional Neural Networks. Advances In Neural Information Processing Systems, 1-9. http://doi.org/http://dx.doi.org/10.1016/j.protcy.2014.09.007

[13] Pu, J., Mintz, G. S., Brilakis, E. S., Banerjee, S., Abdel-Karim, A. R. R., Maini, B., ... Maehara, A. (2012). In vivo characterization of coronary plaques: Novel findings from comparing greyscale and virtual histology intravascular ultrasound and near-infrared spectroscopy. European Heart Journal, 33(3), 372-383. http://doi.org/10.1093/eurheartj/ehr387

[14] Simonyan, K. (2013). Deep Inside Convolutional Networks : Visualising Image Classification Models and Saliency Maps arXiv: 1312 . 6034v2 [ cs . CV ] 19 Apr 2014, 1-8.

[15] Sofian, H., Ng, A., Than, J., Mohamad, S., \& Noor, N. M. (2017). Calcification boundary detection in coronary artery using intravascular ultrasound images. In IEEE Region 10 Annual International Conference, Proceedings/TENCON. http://doi.org/10.1109/TENCON.2017.8228344

[16] Sofian, H., Than, J., Ming, C., Mohamad, S., \& Noor, N. M. (2018). Calcification Detection Using Deep Structured Learning in Intravascular Ultrasound Image for Coronary Artery Disease. In 2nd International Conference on BioSignal Analysis, Processing and Systems (ICBAPS 2018).

[17] Szegedy, C., Ioffe, S., Vanhoucke, V., \& Alemi, A. (2016). Inception-v4, Inception-ResNet and the Impact of Residual Connections on Learning, 4278-4284. http://doi.org/10.1016/j.patrec.2014.01.008

[18] Szegedy, C., Liu, W., Jia, Y., Sermanet, P., Reed, S., Anguelov, D., ... Rabinovich, A. (2014). Going deeper with convolutions. In Proceedings of the IEEE Computer Society Conference on Computer Vision and Pattern Recognition (Vol. 07-12-June, pp. 1-9). http://doi.org/10.1109/CVPR.2015.7298594

[19] Szegedy, C., Vanhoucke, V., Ioffe, S., Shlens, J., \& Wojna, Z. (2015). Rethinking the Inception Architecture for Computer Vision. http://doi.org/10.1109/CVPR.2016.308

[20] Taki, A., Najafi, Z., Roodaki, A., Setarehdan, S. K., Zoroofi, R. A., Konig, A., \& Navab, N. (2008). Automatic segmentation of calcified plaques and vessel borders in IVUS images. International Journal of Computer Assisted Radiology and Surgery.

[21] Unal, G., Bucher, S., Carlier, S., Slabaugh, G., Fang, T., \& Tanaka, K. (2008). Shape-driven segmentation of the arterial wall in intravascular ultrasound images. IEEE Transactions on Information Technology in Biomedicine, 12(3), 335-347. http://doi.org/10.1109/TITB.2008.920620

[22] Xi, P., Shu, C., \& Goubran, R. (2018). Abnormality Detection in Mammography using Deep Convolutional Neural Networks. http://doi.org/10.1109/MeMeA.2018.8438639

[23] Yann LeCun, Leon Bettou, Yoshua Bengio, P. H. (1998). Gradient-Based Applied to Recognition Document. Proceeding of the IEEE, 1-46. 